\title{
An axi-symmetric thermo-hydraulic model to better understand spot laser welding
}

\author{
Marc Medale* — Charline Touvrey** — Rémy Fabbro*** \\ * Polytech' Marseille, Lab. IUSTI, UMR 6595 CNRS \\ Université de Provence \\ Technopole de Château-Gombert \\ 5 rue Enrico Fermi, F-13453 Marseille cedex 13 \\ Marc.Medale@polytech.univ-mrs.fr \\ ** CEA, Centre de Valduc, DAM/DFTN \\ F-21120 Is sur Tille \\ Charline.Touvrey@cea.fr \\ *** GERAILP-LALP, UPR CNRS 1578 \\ 16 bis avenue Prieur de la Côte d'Or \\ F-94114 Arcueil cedex \\ Remy.Fabbro@clfa.fr
}

\begin{abstract}
The aim of this study is to better understand the basic mechanisms leading to possible defect occurrence in spot laser welding. For that purpose we have developed a numerical model, which takes into account the key-hole dynamics together with a dedicated energy deposition model featuring the multiple reflection effects. Many experiments have also been achieved enabling us to report several defect classes. The analysis of some of these scenarios have been performed and favourably compared to experiments.

RÉSUMÉ. L'objectif de cette étude vise à mieux comprendre les mécanismes de base à l'origine de l'apparition de défauts en cours de soudage par laser impulsionnel. Dans ce but, nous avons développé un modèle numérique qui tient compte de la dynamique de creusement en mode key-hole, ainsi qu'un modèle de dépôt d'énergie incluant les effets de réflexions multiples. Plusieurs expériences ont permis de mettre en évidence divers types de défauts. Les simulations numériques de certains de ces scénarios ont été effectuées et comparées aux résultats expérimentaux.

KEYWORDS: computational fluid dynamics, coupled heat and fluid flow, spot laser welding, free and moving interface.

MOTS-CLÉS: mécanique des fluides numérique, thermo-hydraulique, soudage laser impulsionnel, surface libre et mobile.
\end{abstract}

DOI:10.3166/REMN.17.795-806 @ 2008 Lavoisier, Paris

REMN - 17/2008. Giens 2007, pages 795 to 806 


\section{Introduction}

Compared to other welding processes spot laser welding offers several advantages in industrial manufacturing. Indeed, localized temperature gradients require weaker global heating and accordingly lower workpiece distortions. Therefore spot laser welding processes are well suited to problems in which the allowed Affected Heat Zone is required to remain as small as possible in the vicinity of the weld joins. Unfortunately, the operating parameters leading to defect-free weld joins are difficult to obtain, owing to a relatively poor understanding of the problem. Consequently, unsafe weld joins are frequently encountered, polluted by micro or macro pores defects.

In order to better understand why and how these defects occur, both experimental and numerical approaches have been used (Girard et al., 2000), (Kaplan et al., 2002). From the computational point of view, at least three major kinds of difficulties have to be overcome to design robust and accurate numerical models well suited to the spotlaser-welding class of problems: i) The laser-beam energy deposition onto a moving and highly deforming interface (the keyhole) requires dedicated numerical models, whenever the gas phase is not ionized (no plasma). Indeed, the laser beam is trapped into the keyhole, and undergoes multiple reflections along the liquid-gas interface. Several models based on Fresnel's laws have been developed to account for the local energy concentration in the lower part of the keyhole (Fabbro et al., 2000), (Amara et al., 2002); ii) The multiphase problem (solid-liquid-gas) is not straightforward to deal with, especially for alloys made up of several metals. Few authors have proposed to solve the incompressible Navier Stokes equations in all phases (solid, liquid and gas) (Ganesh et al., 1997a), (Ganesh et al., 1997b). Nonetheless, the releavance of such models becomes at least questionable in the gas phase where the Mach number could reach values up to 0.5 when the vaporization rate is very high; iii) The algebraic system resulting from the discretization of this class of problems is very stiff (ill-conditioned) and highly non-linear owing to the strong material and geometrical coupling. Therfore it remains up-to-now computationally expensive to solve even in simplified configurations. For these reasons analytical and semi-analytical approaches are still widely used in keyhole dynamics models (Semak et al., 1999), (Solana et al., 1999), (Jouvard et al., 2001), as far as three-dimensional effects could be neglected (Ki et al., 2001).

The paper is organized as follows. The physical and numerical models specific to the single-laser-pulse interaction are first described in section 2. Then, in a third section several results are presented for a simplified Nd:Yag pulse on a TA6V alloy plate. Finally, we discuss the obtained results; give some concluding remarks and future directions on the way to better understand and optimize spot-laser-welding processes.

\section{Physical and numerical models}

The present model has been designed to study the dynamics of a single laser-pulse onto a thin metal plate, in the keyhole welding mode. So, to tackle this problem we have introduced the following set of simplifying assumptions of physical releavance: 
axial-symmetry condition; no ionization of metallic vapours and shield gas throughout the whole transient study; no beam diffusion inside the vapours. Furthermore, the coupled fluid flow and heat transfer model is focused on the condensed phases (solid and liquid), whereas the dispersed ones (metallic vapours and shield gas) have been modelled in the simplest way to enter the model as boundary conditions. The numerical model is built in the finite element framework and takes advantage of a segregated approach (Rabier et al., 2003), (Medale et al., 2004). Indeed, it consists in splitting the whole problem into sub-problems, easier to successively build up (develop and validate) and also to solve. The four stages considered in the present model are the following: i) compute the laser-beam energy deposition; ii) compute the heat transfer in the whole computational domain; iii) compute the fluid flow in the liquid phase, if any; iiii) perform a computational domain update to satisfy the mass conservation in the condensed phases. The resulting solution algorithm consists in consecutively solving these four stages at each time step of the whole transient process.

\subsection{Laser-beam energy deposition model}

Let us first consider at this stage that the computational domain geometry is entirely defined, so are its boundaries. Moreover, for simpler modelling purpose one discretizes the laser-beam into concentric annulii and split their optical path into two parts: before and after their first interaction with the condensed matter. Prior to their first incidence the intensity $I$ of the ray-tubes is modeled by the Gaussian expression of Equation [1] (Duley, 1999):

$$
\begin{array}{r}
I(r, z)=\frac{2 P}{\pi \omega^{2}(z)} \exp \left(\frac{2 r^{2}}{\omega^{2}(z)}\right) \\
\omega(z)=\omega_{0} \sqrt{1+\left(\frac{z}{z_{R}}\right)^{2}}
\end{array}
$$

where $(r, z)$ designate the axial-symmetry coordinates, $P, \omega$ and $z_{R}$ are the laser beam power, waist and Rayleigh distance, respectively.

Then, based on Fresnel's law, for each ray-tube leaving the beam, one can determine its whole optical path throughout multiple reflections, if any, along the keyhole. At each ray interaction with the condensed matter, one part of the incident optical power is absorbed into the condensed matter, whereas the remaining part is reflected with an angle equal to the incidence one with respect to the outward normal. Indeed, for rather low power density $\mathrm{Nd}$ :Yag laser applications as in the present case, the metallic vapors remain so poorly ionised that one can assume they are perfectly transparent to laser rays, unlike for higher power density or shorter wavelength cases as for $\mathrm{CO}_{2}$ lasers. Therefore the absorbtivity $A$ can be assumed to only depend on both the incidence angle $\alpha$ and temperature $T$ at the incident location. So, the absorbed intensity along the liquid-gas interface corresponds to the sum of the energy density 
transmitted to the condensed matter at each local interaction. It is accounted for in the present model according to Eq. [3] and acts in the heat transfer model as an applied heat flux condition along the interface boundary subset exposed to the laser beam.

$$
q_{l b}(r, z)=\sum_{r a y=1}^{n_{\text {ray }}} \sum_{\text {reflec }=1}^{n_{\text {reflec }}} I(r, z) A(\alpha, T)
$$

\subsection{Heat transfer model}

The macroscopic model dedicated to the condensed phases is derived from the two-phase model (Bennon et al., 1987) and (Ni et al., 1995), in which the representative control volume could contain either purely solid, liquid or a mixture of both phases. Therefore, the conservation equations of mass, momentum and energy apply on the whole condensed matter domain with physical properties specific to each phase. In the present case, the applied heat flux density induces solid-liquid and liquid-vapor phase changes, which are taken into account in the present heat transfer model thanks to an enthalpy formulation of the energy conservation equation [4]:

$$
\frac{\partial \rho_{i}\left(C p_{i} T+\Delta h\right)}{\partial t}+(\vec{V}-\vec{W}) \cdot \vec{\nabla}\left(\rho_{i}\left(C p_{i} T+\Delta h\right)\right)=\nabla \cdot\left(k_{i} \nabla T\right)
$$

where the subscript $i$ designates either solid, mushy or liquid phase; $\rho, C p$ and $k$ stand for the density, specific heat and thermal conductivity, respectively, and $T$ is the temperature. Moreover, as the computational domain has deforming shape and boundaries, the conservation equations are written in an Arbitrary-Lagrangian-Eulerian formulation ( $\vec{V}$ and $\vec{W}$ designate the fluid and mesh velocity vectors, respectively). Concerning the solid-liquid phase change latent enthalpie (denoted $\Delta h$ ), various phenomenological relationships can be used depending on the physical knowledge of the considered alloy. In the present work, it is related to the liquid fraction by the classical linear relationship. On the other hand, the liquid-gas phase change enters the present model as a boundary condition to satisfy the energy conservation equation.

The considered thermal boundary conditions associated with the heat transfer problem can be splitted into four parts. The first one is associated with the axialsymmetry condition (adiabatic condition along the symmetry axis); the second one is the applied heat flux associated with the laser beam interaction ( $q_{l b}$, Equation [3]); the third boundary condition models the rate of thermal energy devoted to vaporization or condensation $q_{l v}$, if any. It is accounted for in our model as a boundary condition along the interface part where liquid-vapor phase change occurs (Equation [5]). The vaporization rate $\dot{m}_{l v}$ is drawn from the mass balance across the liquid-vapor interface, Equation [6].

$$
\begin{array}{r}
q_{l v}=\dot{m}_{l v} \Delta h_{l v} \\
\rho_{l} \vec{V}_{l / \Sigma} \cdot \vec{n}=\rho_{v} \vec{V}_{v / \Sigma} \cdot \vec{n}
\end{array}
$$


Finally, the forth thermal boundary condition is associated with the radiative and convective heat transfer along the other boundaries of the condensed phase domain. They are modeled in the classical way, Equation [7]:

$$
q_{r c}=\sigma \varepsilon\left(T^{4}-T_{\infty}^{4}\right)+h\left(T-T_{\infty}\right)
$$

where $\sigma, \varepsilon$ and $h$ designate the Stefan constant, the emissivity and convective heat transfer coefficients, respectively, while $T_{\infty}$ is the room temperature.

\subsection{Fluid flow model}

As far as melting and vaporization are involved in the process, the heat transfer in the melted pool is significantly dominated by advection. Therefore, one models the liquid metal flow as an incompressible fluid flow and the classical Navier-Stokes equations should be supplemented with an extra term to account for the smooth solid-liquid transition characteristic to metal alloys. Moreover, in the solidification zones an interdendrite fluid flow could also exist and should be modelled at the macroscopic scale (Voller et al., 1987). Consequently, the mass and momentum conservation equations here again in an ALE framework read, Equation $[8,9]$ :

$$
\begin{array}{r}
\vec{\nabla} \cdot \vec{V}=0 \\
\rho_{l}\left(\frac{\partial \vec{V}}{\partial t}+(\vec{V}-\vec{W}) \cdot \overline{\bar{\nabla}} \vec{V}\right)=\nabla \cdot \overline{\bar{\sigma}}+\overrightarrow{f_{v}}+\overrightarrow{S_{u}}
\end{array}
$$

The first constitutive law used to close the preceding set of equations is that of a Newtonian fluid (Equation [10]). Secondly, the Boussinesq approximation (Equation [11]) is used to take into account buoyancy in the incompressible fluid flow model. Finally, the last term is selectively introduced in the mushy zone to model at the macroscopic scale the fluid flow which takes place at microscopic scale in the vicinity of the solid-liquid interface. It is modelled as a Darcy-like flow (Equation [12]), which furthermore ensures a vanishing velocity when approaching the solid metal without any explicit front tracking technique.

$$
\begin{array}{r}
\overline{\bar{\sigma}}=-p \overline{\bar{I}}+\mu\left[\overline{\bar{\nabla}} \vec{V}+(\overline{\bar{\nabla}} \vec{V})^{t}\right] \\
\overrightarrow{f_{v}}=\rho_{l_{0}}\left[1-\beta\left(T-T_{0}\right)\right] \vec{g} \\
\overrightarrow{S_{u}}=\frac{\mu}{K}(\vec{V}-\vec{W})
\end{array}
$$

where $\mu, \beta$ and $K$ are the liquid metal dynamic viscosity, thermal expansion coefficient and permeability of the porous media in the mushy zone, respectively. 
The boundary conditions that apply to the liquid metal fluid flow problem read in a formal notation, Equation [13, 14]:

$$
\begin{aligned}
\vec{V}=\vec{W} & =\overrightarrow{0} \text { on } \partial \Omega_{s l} \\
\vec{T}_{l}+\vec{T}_{g}+\frac{\gamma}{R} \vec{n}+\frac{\partial \gamma}{\partial s} \overrightarrow{t_{g}} & =\overrightarrow{0} \text { on } \partial \Omega_{l g}
\end{aligned}
$$

Indeed, the stresses acting along the liquid-gas interface $\vec{T}_{i}(i=l, g)$ result from the interactions between the liquid and gas phases, which can be attributed to: i) the recoil pressure associated with the evaporation process; ii) the shear stress induced by the high velocity metallic vapours flowing out from the key hole; iii) surface tension effects: capillary forces owing to high curvature of the liquid-gas interface $(\gamma$ is the surface tension coefficient, $R$ the interface radius of curvature) and thermo-capillary forces along the interface induced by surface tension gradients $\left(\frac{\partial \gamma}{\partial s}\right)$.

\subsection{Computational domain update - Mesh adaption model}

As the computational domain is restricted to the condensed matter (solid and liquid phases), it evolves and deforms in the course of the computation. So, to prevent from excessive mesh distorsion responsible for poor accuracy in the solution, we supplement our numerical model with a steady pseudo-elastic problem for the mesh at each time step, Equation [15], (Rabier et al., 2003). It is formulated in nodal mesh displacement variables $\vec{U}_{m}$, throughout an elastic-like stress-strain relationship, Equation [16], in which $E_{m}$ and $\nu_{m}$ are numerical coefficients tuned to produce satisfactory mesh quality.

$$
\begin{array}{r}
\nabla \cdot \overline{\bar{\sigma}}_{m}=\overrightarrow{0} \\
\overline{\bar{\sigma}}_{m}=\frac{E_{m}}{1+\nu_{m}} \overline{\bar{\varepsilon}}_{m}+\frac{E_{m} \nu_{m}}{\left(1+\nu_{m}\right)\left(1-2 \nu_{m}\right)} \operatorname{tr}\left(\overline{\bar{\varepsilon}}_{m}\right) \overline{\bar{I}} \\
\overline{\bar{\varepsilon}}_{m}=\frac{1}{2}\left[\overline{\bar{\nabla}} \vec{U}_{m}+\left(\overline{\bar{\nabla}} \vec{U}_{m}\right)^{t}\right]
\end{array}
$$

The mass conservation of the condensed matter requires to explicitly satisfy a kinematic boundary condition along the computational domain boundary, which results in a vanishing normal component of the relative velocity between the liquid fluid flow and the mesh along the boundaries, Equation [18].

$$
\vec{U}_{m} \cdot \vec{n}=\Delta t \vec{V} \cdot \vec{n} \text { on } \partial \Omega_{m}
$$

Once the solution of the pseudo-elastic problem is obtained one performs the update of the mesh node coordinates $\vec{X}_{m}$ according to Equation [19]:

$$
\vec{X}_{m}^{t+\Delta t}=\vec{X}_{m}^{t}+\vec{U}_{m}
$$




\section{Numerical model verifications and validations}

As a first step of verification, we have tested separately each component of the global model, which represents one of the main advantages of the chosen segregated approach. The thermo-hydraulic model has already been validated previously in both capillary and gravity free surface problems (Rabier et al., 2003), so one focusses here on the laser-beam energy deposition model. Indeed, in the keyhole mode configuration the laser beam undergoes multiple reflections, so one want first to check the model in a given and fixed keyhole shape. The sinusoidal shape has been selected as a test case in a fixed geometry because it exhibits several focus zones where spatial resolution could be crucial. The reflected rays in the sinusoidal shape keyhole are displayed in Figure 1, together with the resulting dimensionless heat flux along the curvilinear abscissa. One can observe the very sharp peaks in the dimensionless heat flux density plot, which result from the two focus zones in the lower part of the keyhole. In order to assess the solution accuracy in such a configuration we have performed a convergence study and sensibility analysis of the numerical integration parameters (spatial resolution along the keyhole boundary, number of integration points per finite element, number of rays to discretize the laser beam) and we present in Table 1 the results. It turns out that in this keyhole shape one needs at least five rays per element and fifteen integration points per ray in order to find out the converged results. So these values will be used as minimum values to set up the user defined parameters in the computations.

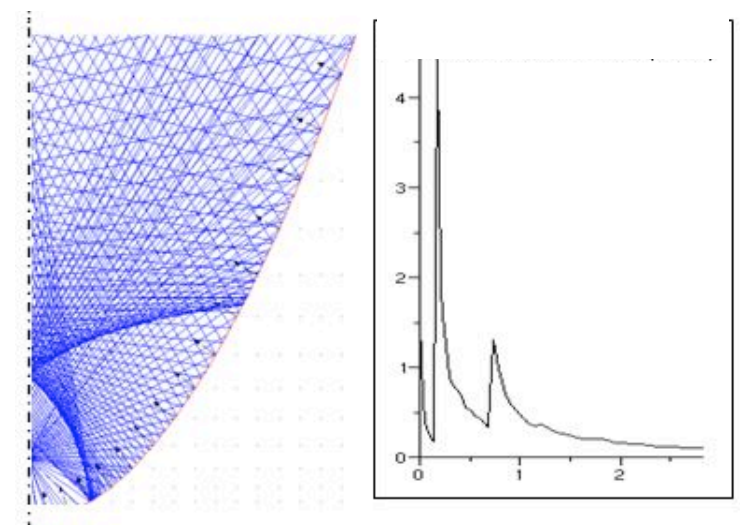

Figure 1. Energy deposition in a keyhole of sinusoidal shape. Reflected rays (left) and dimensionless heat flux along the curvilinear abscissa (right)

\section{Analysis}

We consider a single laser pulse carried out in the middle of a $3 \mathrm{~mm}$ metal sheet (no junction zone), to satisfy the axial-symmetry condition. The delivered laser beam power is considered constant $(P=1500 \mathrm{~W})$ throughout the $10 \mathrm{~ms}$ laser pulse duration. It is carried by an optical fiber and its spatial distribution over the metal sheet 
Table 1. Spatial convergence analysis and sensibility to numerical integration parameters. $N_{1}, N_{2}, N_{3}, q_{p 1}$ and $q_{p 2}$ stand for the number of integration points per ray, number of integration points per element, number of rays per element, first peak intensity, second peak intensity, respectively

\begin{tabular}{|c|c|c|c|c|}
\hline $\mathrm{N}_{1}$ & $\mathrm{~N}_{2}$ & $\mathrm{~N}_{3}$ & $\mathrm{q}_{p 1}$ & $\mathrm{q}_{p 2}$ \\
\hline 1 & 1 & 2 & 2.54 & 1.10 \\
\hline 1 & 3 & 4 & 3.05 & 1.23 \\
\hline 1 & 9 & 10 & 3.90 & 1.42 \\
\hline 1 & 19 & 20 & 4.36 & 1.43 \\
\hline 1 & 49 & 50 & 4.36 & 1.43 \\
\hline 20 & 40 & 3 & 2.94 & 1.16 \\
\hline 20 & 60 & 4 & 3.69 & 1.22 \\
\hline 15 & 60 & 5 & 4.36 & 1.43 \\
\hline
\end{tabular}

is assumed to be a $600 \mu \mathrm{m}$ diameter top hat at the focal point. To later on compare computations to experiments carried out at our CEA laboratory, the material properties of TA6V plate (aluminium alloy) have been considered in the computations (see physical properties provided in Table 2).

Table 2. Physical properties used in computations for the TA6V alloy at $1923 \mathrm{~K}$

\begin{tabular}{|l|rl|}
\hline Melting temperature & 1923 & $\mathrm{~K}$ \\
\hline Solid-liquid latent enthalpy & $410^{5}$ & $\mathrm{~J} . \mathrm{kg}^{-1}$ \\
\hline Vaporization temperature & 3591 & $\mathrm{~K}$ \\
\hline Liquid-gas latent enthalpy & $8.810^{5}$ & $\mathrm{~J} . \mathrm{kg}^{-1}$ \\
\hline Liquid density & 4110 & $\mathrm{Kg} \cdot \mathrm{m}^{-3}$ \\
\hline Dynamic viscosity of liquid & $3.410^{-3}$ & $\mathrm{~Pa} . \mathrm{s}$ \\
\hline Surface tension coefficient & 1.6 & $\mathrm{~N} . \mathrm{m}^{-1}$ \\
\hline Thermal coef. surface tension & $310^{-4}$ & $\mathrm{~N} . \mathrm{m}^{-1} . \mathrm{K}^{-1}$ \\
\hline Specific heat & 743 & $\mathrm{~J} . \mathrm{kg}^{-1} \cdot \mathrm{K}^{-1}$ \\
\hline Thermal conductivity & 35 & $\mathrm{~W} . \mathrm{m}^{-1} . \mathrm{K}^{-1}$ \\
\hline
\end{tabular}

The thermo-hydraulic finite element model has been previously validated in simplified configurations (Rabier et al., 2003), (Medale et al., 2004). So, the present study aims to validate the energy deposition model on a dynamically deforming keyhole where multiple reflections take place. As the free surface deforms to a large extent (keyhole) during the laser pulse, the laser energy deposition becomes of first concern, especially when multiple reflections occur in the keyhole. The computational domain is $3 \mathrm{~mm}$ thick (metal sheet thickness) and $1 \mathrm{~mm}$ width, initially at room temperature $\mathrm{T}_{0}=300 \mathrm{~K}$. In the present computations we assume constant physical properties (given in Table 2) all over the solid-liquid domain, and the simplified thermal heat 
exchange boundary conditions (Equation [7]) are set to: $\varepsilon=0.5, h=10 \mathrm{Wm}^{-1} \mathrm{~K}^{-1}$ and $T_{\infty}=300 \mathrm{~K}$. The mesh used is made up of $75 \times 150$ bi-quadratic quadrilateral finite elements (Q9), non-uniformly distributed in the radial and axial directions. A constant time increment of $\Delta t=10^{-2} \mathrm{~ms}$ has been used, so the computation of the whole laser pulse requires 1000 time steps.

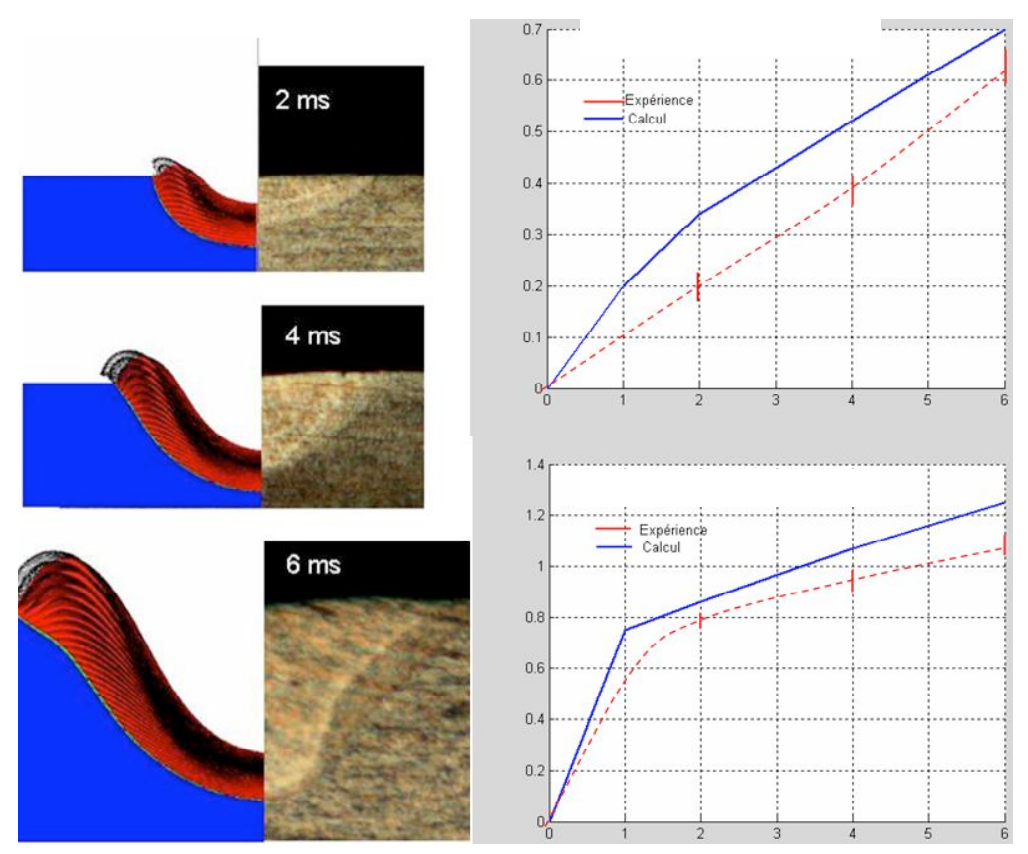

Figure 2. Comparison between computations and experiments. Melted pool and affected heat zone (left); keyhole depth (top right) and width (bottom right) plots versus time in ms. Solid line: computations; dashed line: experiements

The recoil pressure applied onto the free surface induces a fast drilling of the keyhole. The computations faithfully reproduce this behaviour, as shown in Figure 2 for $t=2,4,6 \mathrm{~ms}$, respectively. As the keyhole becomes deeper and deeper the number of laser-rays reflections increases significantly, as can be observed in Figure 3, which depicts a close up of the reflected ray paths in the vicinity of the keyhole tip, together with the corresponding thermal field, at time $t=5 \mathrm{~ms}$. The multiple reflections inside the highly deformed keyhole result in a noticeable power intensity focus over the keyhole tip. Indeed the global absorbtivity coefficient, which measures the energy efficiency of the interaction, raises from roughly $50 \%$ at the beginning of the laser pulse up to $80 \%$ as soon as the keyhole is deep enough to trap most of the reflected rays. The finite element mesh adaption technique performs well to deal with the moving boundary problem in such a highly deformed computational domain, as shown in Figure 4 for $t=3,5 \mathrm{~ms}$, respectively, resulting in a fairly good accuracy over the whole computations. 
804 REMN - 17/2008. Giens 2007

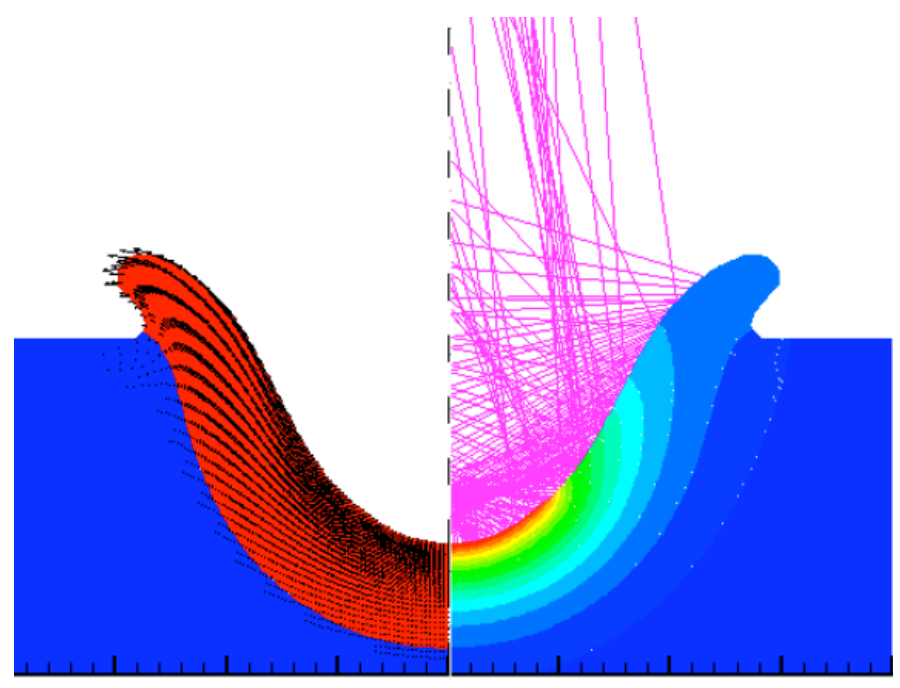

Figure 3. Velocity field in the liquid metal (left), reflected rays at the liquid-gas interface and thermal field (right) at $t=5 \mathrm{~ms}$
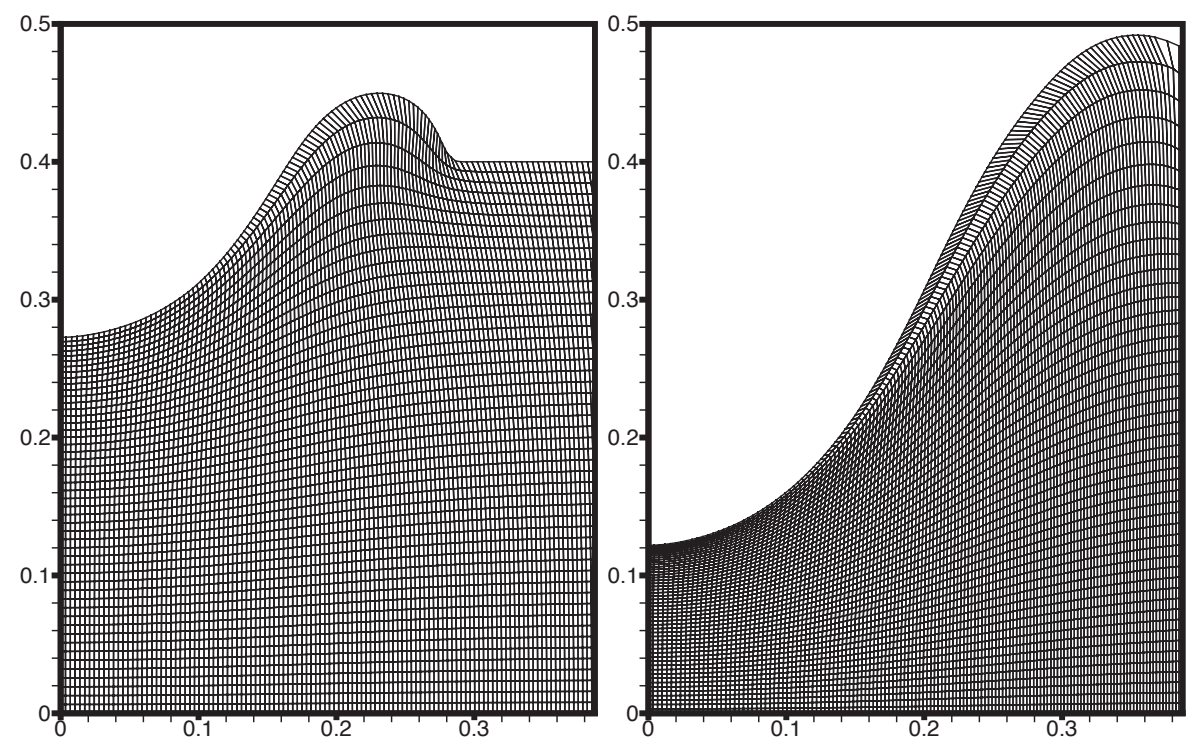

Figure 4. Computational mesh evolution in the course of time. At $t=3 \mathrm{~ms}$ (left) and $t=5 m s$ (right) 


\section{Conclusions}

The numerical model we have developed to study the dynamics of a single laser pulse acting on a thin metal sheet is expected to provide us with a better understanding of the basic mechanisms that could be responsible for numerous defects in industrial spot laser welding processes. It is focussed on the condensed phases (solid and liquid), but take into account the dispersed phase (vapors and shield gas) in a simplified manner. It is made up of four main stages: the laser energy deposition, the heat transfer in computational domain, the fluid flow in the liquid phase, if any, and finally a computational domain and mesh update to account for the total mass conservation and large distorsion of the liquid-gas interface (keyhole). The present study deals with an advanced level validation whose objective was the test and verification of the energy deposition model on a dynamically deforming keyhole where multiple reflections take place. As the free surface shape evolves continually during the laser pulse, the ray paths (determined by Fresnel's law) become of first concern to accurately compute the laser energy deposition, especially when multiple reflections occur in the keyhole. The comparison of the computational results to the available lab experiements are in qualitatively good agreement, despite a finer mesh should be considered in order to faithfully take into account both solid-liquid and liquid-gas phase changes. Indeed, a time shift has been observed in the computations, which is probably due to loose time integration of the energy conservation equation.

The next step in the spot laser welding modelling will be to extend the present model to full three dimensional geometry in order to be able to represent the actual welding process, which consist in successive laser impacts along the joining line.

\section{References}

Amara E., Bendib A., “ Modelling of vapour flow in deep penetration laser welding”, J. Phys. D: Appl. Phys., vol. 35, p. 272-280, 2002.

Bennon W., Incropera F., “ A continuum model for momentum, heat and species transport in binary solid-liquid phase change systems - 1. Model formulation, 2. Application to solidification in a rectangular cavity", Int. J. Heat Mass Transf., 1987.

Duley W., Laser Welding, Wiley Interscience, 1999.

Fabbro R., Chouf K., “ Keyhole modelling during laser welding”, J. Phys. D: Appl. Phys., vol. 87, p. 4075-4083, 2000.

Ganesh R., Faghri A., Hahn Y., “ A generalized thermal modelling for laser drilling process I. Mathematical modelling and numerical methodology", J. Heat Mass Transfer, vol. 40, p. 3351-3360, 1997a.

Ganesh R., Faghri A., Hahn Y., “ A generalized thermal modelling for laser drilling process II. Numerical simulation and results", J. Heat Mass Transfer, vol. 40, p. 3361-3373, 1997b.

Girard K., Jouvard J., Boquillon J., Bouilly P., Naudy P.in S. Bellingham (ed.), SPIE Conference, vol. 3888, p. 418-428, 2000. 
Jouvard J., Girard K., Perret O., “ Keyhole formation and power deposition in ND:YAG laser spot welding”, J. Phys. D: Appl. Phys., vol. 34, p. 364-372, 2001.

Kaplan A., Mizutani M., Katayama S., Matsunawa A., “ Unbounded keyhole collapse and bubble formation during pulsed laser interaction with liquid zinc", J. Phys. D: Appl. Phys., vol. 35, p. 1218-1228, 2002.

Ki H., Mohanty P., Mazumder H., “ Modelling of high density laser material interaction using fast level set method”, J. Phys. D: Appl. Phys., vol. 34, p. 364-372, 2001.

Medale M., Rabier S., Xhaard C., “ A thermo-hydraulic numerical model for high energy welding processes", Rev. Eur. Elements Finis, vol. 13, p. 207-229, 2004.

Ni J., Incropera F., “ Extension of the continuum model for transport phenomena occuring during metal alloy solidification - 1 . The conservation equations, 2. Microscopic considerations", Int. J. Heat Mass Transf., vol. 38, p. 1271-1296, 1995.

Rabier S., Medale M., “ Computation of free surface flows with a projection FEM in a moving mesh framework”, Comput. Methods Appl. Mech. Engrg., vol. 192, p. 4703-4721, 2003.

Semak W., Bragg W., Damkroger B., Kempkas S., “ Temporal evolution of the temperature field in the beam interaction zone during laser-material processing", J. Phys. D: Appl. Phys., vol. 32, p. 1819-1825, 1999.

Semak W., Matsunawa A., " The role of recoil pressure in energy balance during laser - materials processing”, J. Phys. D: Appl. Phys., vol. 30, p. 2541-2552, 1997.

Solana P., Kapadia P., Dowden J., Marsden P., “ An analytical model for laser drilling of metals with absorption within the vapour”, J. Phys. D: Appl. Phys., vol. 32, p. 942-952, 1999.

Voller V., Prakash C., " A fixed grid numerical modelling methodology for convection diffusion mushy region phase change problems", Int. J. Heat Mass Transf., vol. 24, p. 1709-1718, 1987. 